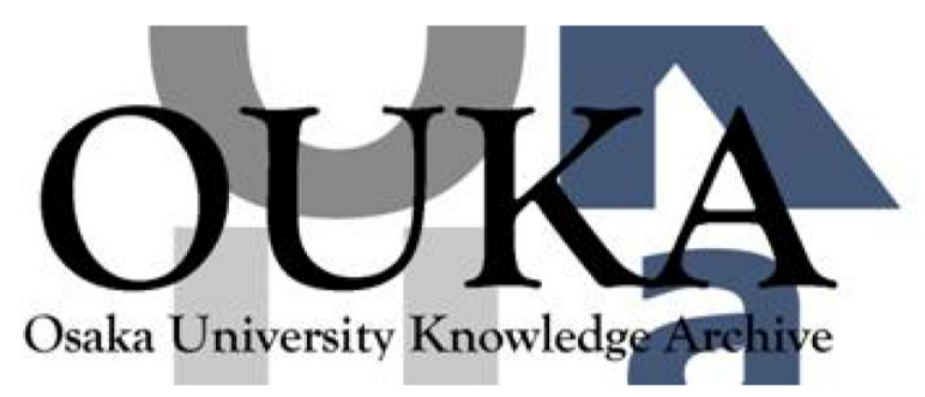

\begin{tabular}{|c|l|}
\hline Title & $\begin{array}{l}\text { Optical properties, spectral narrowing of } \\
\text { photoluminescence and blue electroluminescence } \\
\text { of poly(phenylene pyridine) derivatives }\end{array}$ \\
\hline Author(s) & Fuji i, A.; Ootake, R.; Fujisawa, T. et al. \\
\hline Citation & Applied Physics Letters. 77(5) p. 660-p. 662 \\
\hline Issue Date & $2000-07-27$ \\
\hline oaire:version & VoR \\
\hline URL & https://hdl. handle. net/11094/75654 \\
\hline rights & \\
\hline Note & \\
\hline
\end{tabular}

Osaka University Knowledge Archive : OUKA

https://ir. Library. osaka-u. ac. jp/

Osaka University 


\section{Optical properties, spectral narrowing of photoluminescence and blue electroluminescence of poly(phenylene pyridine) derivatives}

Cite as: Appl. Phys. Lett. 77, 660 (2000); https://doi.org/10.1063/1.127077

Submitted: 24 April 2000 . Accepted: 08 June 2000 . Published Online: 27 July 2000

A. Fujii, R. Ootake, T. Fujisawa, M. Ozaki, Y. Ohmori, Tong Laga, K. Yoshino, H.-F. Lu, H. S. O. Chan, and S.C. $\mathrm{Ng}$

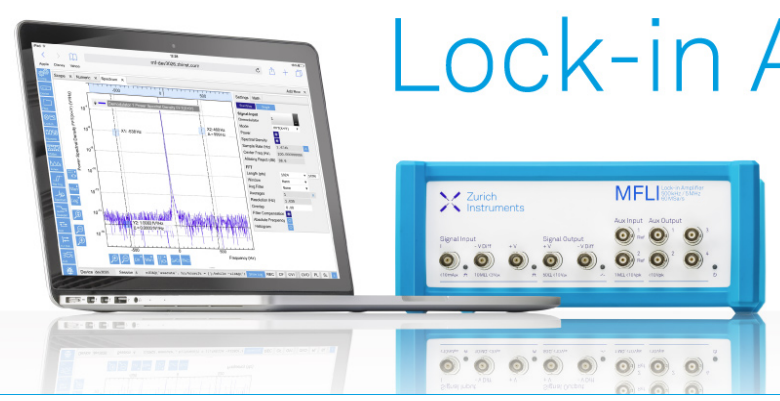

Amplifiers

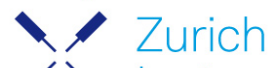

- Instruments

Watch the Video 


\title{
Optical properties, spectral narrowing of photoluminescence and blue electroluminescence of poly(phenylene pyridine) derivatives
}

\author{
A. Fujii, R. Ootake, T. Fujisawa, M. Ozaki, Y. Ohmori, Tong Laga, ${ }^{\text {a) }}$ and K. Yoshino ${ }^{\text {b) }}$ \\ Department of Electronic Engineering, Graduate School of Engineering, Osaka University, \\ 2-1 Yamada-oka, Suita, Osaka 565-0871, Japan \\ H.-F. Lu, H. S. O. Chan, and S.-C. Ng \\ Department of Chemistry, National University of Singapore, 119260, Singapore
}

(Received 24 April 2000; accepted for publication 8 June 2000)

\begin{abstract}
Optical properties of poly(phenylene pyridine) derivatives, poly(2,5-dialkoxy-1,4phenylenealt-2,5-pyridine) (PHOnPY25), such as optical absorption, photoluminescence (PL), and electroluminescence (EL), have been studied. The electronic energy structures of PHOnPY25 have been determined by optical and electrochemical measurements. Strong PL with high quantum efficiency has been clarified in the films, and spectral narrowing has been observed by pulse excitation of a nitrogen laser. Intense blue EL has also been demonstrated. (C) 2000 American Institute of Physics. [S0003-6951(00)04031-6]
\end{abstract}

The use of $\pi$-conjugated conducting polymers as functional materials has attracted much attention from the point of view of basic science and applications, because of their high processability, stability, and promising electrical and optical properties. ${ }^{1,2}$ Among various conducting polymers, alkyl- or alkoxy-substituted polymers, such as poly(3alkylthiophene $)^{3} \quad$ poly(9,9-dialkylfluorene) (PDAF), ${ }^{4,5}$ poly(p-phenylene) (PPP) derivative, ${ }^{6}$ and poly(2,5-dialkoxyp-phenylene vinylene $)^{7,8}$ are the most attractive materials, because of their high solubility in common organic solvents, their fusibility at relatively low temperatures, and their high luminescent quantum efficiency.

Utilizing conducting polymers with relative large band gap, polymer blue light-emitting diodes (LED) could be realized. We have demonstrated the blue LEDs by utilizing PDAF, ${ }^{5}$ PPP derivative, ${ }^{6}$ or polyacetylene derivative ${ }^{9}$ as emission layer.

On the other hand, alternating copolymers with a couple of $\pi$-conjugated segments in each monomer unit of polymer main chain are expected to possess unique characteristics and functionalities. Recently, such an alternating copolymer with 2,5-thienylene and 1,4-phenylene units, namely, regioregular poly(3-butyl-2,5-thienylene-alt-1,4-phenylene), was synthesized and demonstrated the solubility in common solvents and the green electroluminescent properties. ${ }^{10}$ Alternating copolymers synthesized by the modified synthetic process should also have a possibility to demonstrate the blue luminescent properties.

In this letter, we report the optical properties, such as photoluminescence (PL) and spectral narrowing, and the electroluminescent properties of alternating copolymer poly(2,5-dialkoxy-1,4-phenylene-alt-2,5-pyridine) (PHOnPY25).

In this study, we used four kinds of PHOnPY25 with different alkoxy side chain length $(n=4,8,12,16)$, which

\footnotetext{
${ }^{a)}$ Present address: Inner Mongolia Nationality Normal College, Tong Liao, Inner Mongolia, China.

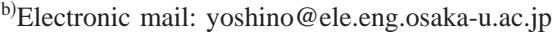

were synthesized by a method reported elsewhere. ${ }^{11}$ These polymers, the molecular structures of which are shown in the inset of Fig. 1, have 2,5-dialkoxy-1,4-phenylene and 2,5pyridine in a monomer unit. The polymers are soluble in common organic solvents such as chloroform.

Thin films of the polymers were formed on quartz plates and In-Sn-oxide (ITO)-coated glass plates with a sheet resistance of $10 \Omega / \square$ by a spin-coating method for measurements. Absorption and PL were measured at room temperature in an evacuated quartz vessel using a Hewlett Packard HP8452A spectrophotometer and a Hitachi F-2000 spectrophotometer, respectively.

Electrochemical measurements, such as cyclic voltammetry, were carried out with a three-electrode system consisting of a working electrode with the sample, a platinum counter electrode, and a silver reference electrode in an electrolytic solution of tetrabutylammonium tetrafluoroborate/ acetonitrile using a Hokuto Denko HB-105 function generator and the HA-105 potentiostat.

PL quantum efficiency was measured by a system consisting of an $\mathrm{Ar}^{+}$laser, a mechanical light chopper, an integrated sphere with photodiode, and a lock-in amplifier.

For the PL measurements at high excitation intensities, we used a laser beam with 600 ps pulses with the energy per

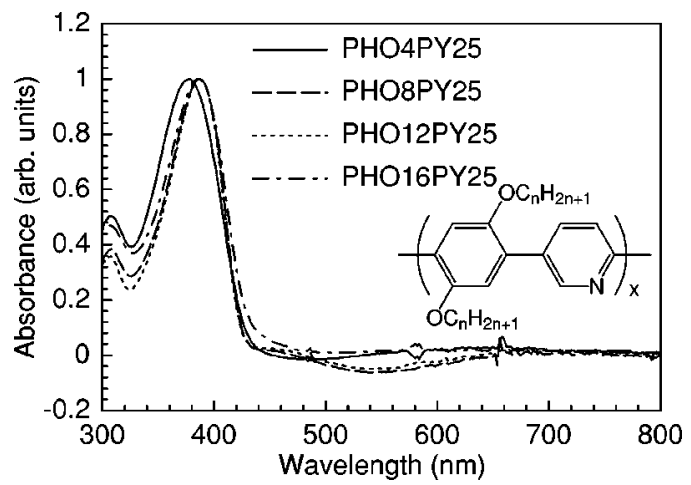

FIG. 1. Absorption spectra of PHOnPY25. Inset shows the molecular structure of PHOnPY25. 


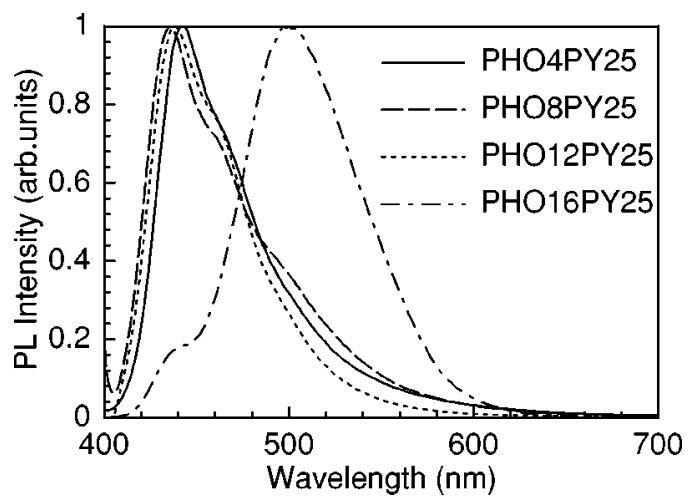

FIG. 2. PL spectra of PHOnPY25.

pulse ranging from 1 to $110 \mu \mathrm{J}$, provided by a nitrogen laser, the wavelength of which was $337.1 \mathrm{~nm}$. The pump laser beam was focused by a cylindrical lens onto the PHOnPY25 film and the emission from the side of the film was detected by a photonic multichannel analyzer (PMA-11, Hamamatsu Photonics Co.).

LEDs with the structure of ITO/PHOnPY25/Al were fabricated by vacuum deposition of $\mathrm{Al}$ onto thin polymer films on ITO glass substrates. Electrical properties such as current-voltage characteristics and electroluminescence (EL) spectrum were measured at liquid nitrogen temperature (77 K) using conventional methods, which were described in detail previously. ${ }^{3}$

Figure 1 also shows the absorption spectra of PHOnPY25. As evident from this figure, the absorption edges and peaks are almost the same for all of the PHOnPY25. That is, the absorption edge is not influenced by the length $(n)$ of the substituted alkoxy moieties. From an analysis of the absorption spectral edge corresponding to the highest occupied molecular orbital (HOMO) - the lowest unoccupied molecular orbital gap of the PHOnPY25, using the relationship $(h \nu \times \alpha)^{2}$ vs h $\nu(\alpha$ : absorption coefficient, $h$ : Planck's constant, $\nu$ : frequency) under the assumption of direct transition, the band gap energies of PHOnPY25 are evaluated as $3.0 \mathrm{eV}$, which are independent of the alkoxy substituent length. The interchain interaction might be quite small in the case of the polymers.

The top of the valence band, that is, the HOMO, was determined from the observed threshold potential of the electrochemical oxidation in the cyclic voltammogram of PHOnPY25. The threshold potentials of PHOnPY25 are estimated to be about $1.3 \mathrm{eV}$ below the work function of silver.

When the PHOnPY25 films were excited by violet light at $385 \mathrm{~nm}$ from a Xe lamp, which was corresponding to the absorption peak wavelength, strong blue PL was observed. The spectra of PHOnPY25 are shown in Fig. 2. The peak wavelengths are located at 430-440 $\mathrm{nm}$ for PHO4PY25, PHO8PY25, and PHO12PY25, and at $500 \mathrm{~nm}$ for PHO16PY25. PL quantum efficiencies of the polymer films are evaluated to be about $20 \%$, when the laser line at 363.7 $\mathrm{nm}$ of an $\mathrm{Ar}^{+}$laser is adopted as an excitation source. Such efficiency is comparable with previously reported efficiency of blue luminescent conducting polymers. ${ }^{12}$

When the excitation intensity of the nitrogen laser pulse was increased, the emission spectra of PHO12PY25 film changed as shown in Fig. 3. It is seen that the broad PL

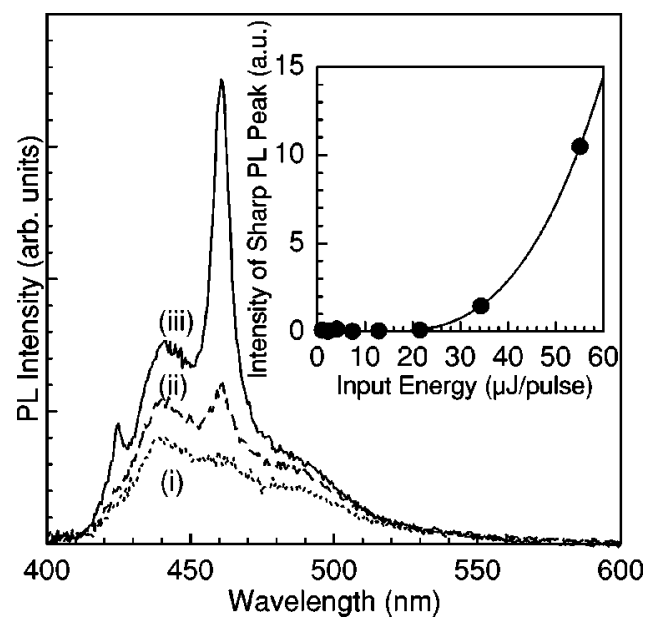

FIG. 3. Optical emission spectra at different excitation pulse energies in PHO12PY25 thin film. The excitation pulse energies were 43.1 (i), 68.6 (ii), 110.2 (iii), respectively. Inset shows the emission peak intensity dependence on the excitation intensity in PHO12PY25 thin film.

spectrum obtained at low excitation intensities changes at high excitation intensities to a much narrower and stronger emission band peaked at $460 \mathrm{~nm}$ with the spectral width of 5 $\mathrm{nm}$. The emission spectral narrowing is accompanied with a nonlinear amplification as illustrated in the inset of Fig. 3, the PL intensities of which are subtracted by the linearly increasing factor of PL intensities. As apparent in Fig. 3, the sharp peak starts to be observed at the input energy of 20 $\mu \mathrm{J} /$ pulse and the intensity of the peak at $460 \mathrm{~nm}$ changes its dependence on excitation intensity to superlinear. The spectrally narrowed emission is interpreted to be caused due to the amplified spontaneous emission ${ }^{12-14}$ enhanced by the optical waveguiding in the polymer film at this stage. It should also be noticed that nitrogen laser are available as a short pulse excitation source and that the simple measurement systems with nitrogen lasers might be appropriate for such optical measurements.

In the case of LEDs utilizing PHOnPY25 as emission layers, that is, with a structure of ITO/PHOnPY25/Al, strong blue EL was observed. The LEDs exhibit typical rectifying characteristics. That is, in this case, ITO and Al layers act as hole and electron injecting electrodes, respectively. The emission intensity starts to increase at around $15 \mathrm{~V}$. The driving voltages tended to increase with increasing polymer thickness. The emission intensity increased monotonically with increasing injection current.

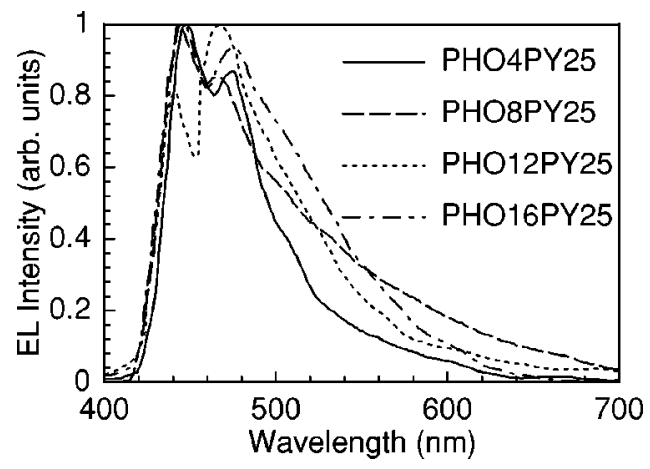

FIG. 4. EL spectra of PHOnPY25 at $77 \mathrm{~K}$. 
By applying a positive bias voltage of, for example, 20 $\mathrm{V}$ to the PHOnPY25 LEDs, a bright blue emission was obtained, the EL spectra of which is shown in Fig. 4. The emission peak wavelengths of PHOnPY25 are around 430$440 \mathrm{~nm}$, which coincide with those of PL.

Although some alternating copolymers have already been known as EL materials, ${ }^{10}$ such blue luminescent alternating copolymers with high quantum efficiencies have hardly been reported. It should be emphasized that alternating copolymers, such as PHOnPY25, could be candidates of blue luminescent materials for organic blue LEDs and blue lasers. Detailed studies are now in progress to clarify the emission mechanism of PHOnPY25.

In conclusion, the optical properties of PHOnPY25, such as absorption spectra, PL, spectral narrowing and EL, were studied. The electronic energy structures of PHOnPY25 were determined by optical and electrochemical measurements. Spectral narrowing of PL was observed from PHOnPY25 films by the pulse excitation of the nitrogen laser. Intense blue EL was also demonstrated in the LEDs of PHOnPY25.

This work was partly supported by a Grant-in-Aid for Scientific Research from the Ministry of Education, Science, Sports and Culture.
${ }^{1}$ E. M. Conwell, in Handbook of Organic Conductive Molecules and Polymers, edited by H. S. Nalwa (Wiley, New York, 1997), Vol. 4, Chap. 1,

p. 1.

${ }^{2}$ Handbook of Conducting Polymers, edited by T. A. Skotheim, R. L. Elsenbaumer, and J. R. Reynolds (Marcel Dekker, New York, 1998).

${ }^{3}$ Y. Ohmori, M. Uchida, K. Muro, and K. Yoshino, Jpn. J. Appl. Phys., Part 2 30, L1938 (1991).

${ }^{4}$ M. Fukuda, K. Sawada, and K. Yoshino, Jpn. J. Appl. Phys., Part 2 28, L1433 (1989).

${ }^{5}$ Y. Ohmori, M. Uchida, K. Muro, and K. Yoshino, Jpn. J. Appl. Phys., Part 2 30, L1941 (1991).

${ }^{6}$ M. Hamaguchi, H. Sawada, J. Kyokane, and K. Yoshino, Chem. Lett., 527 (1996).

${ }^{7}$ M. Hamaguchi and K. Yoshino, Jpn. J. Appl. Phys., Part 2 33, L1478 (1994).

${ }^{8}$ M. Hamaguchi and K. Yoshino, Jpn. J. Appl. Phys., Part 2 34, L712 (1995).

${ }^{9}$ M. Hirohata, K. Tada, R. Hidayat, T. Masuda, and K. Yoshino, Jpn. J. Appl. Phys., Part 2 36, L302 (1997).

${ }^{10}$ S. C. Ng, J. M. Xu, H. S. O. Chan, A. Fujii, and K. Yoshino, J. Mater. Chem. 9, 381 (1999).

${ }^{11}$ S. C. Ng, H.-F. Lu, H. S. O. Chan, A. Fujii, T. Laga, and K. Yoshino, Adv. Mater. (to be published).

${ }^{12}$ M. N. Shkunov, R. Österbacka, A. Fujii, K. Yoshino, and Z. V. Vardeny, Appl. Phys. Lett. 74, 1648 (1999).

${ }^{13}$ S. V. Frolov, W. Gellermann, M. Ozaki, K. Yoshino, and Z. V. Vardeny, Phys. Rev. Lett. 78, 729 (1997).

${ }^{14}$ S. V. Frolov, M. Shkunov, Z. V. Vardeny, K. Tada, R. Hidayat, M. Hirohata, M. Teraguchi, T. Masuda, and K. Yoshino, Jpn. J. Appl. Phys., Part 2 36, L1268 (1997). 